\title{
On Product Logic with Truth-constants
}

\author{
Petr Savický \\ Institute of Computer Science, Academy of Sciences of the Czech Republic \\ Czech Republic \\ Roberto Cignoli \\ Instituto Argentino de Matemática - CONICET \\ Argentina \\ Francesc Esteva, Lluís Godo, Carles Noguera \\ Institut d'Investigació en Intel-ligència Artificial - CSIC \\ Catalonia, Spain
}

\begin{abstract}
Product Logic $\Pi$ is an axiomatic extension of Hájek's Basic Fuzzy Logic BL coping with the 1-tautologies when the strong conjunction \& and implication $\rightarrow$ are interpreted by the product of reals in $[0,1]$ and its residuum respectively. In this paper we investigate expansions of Product Logic by adding into the language a countable set of truth-constants (one truth-constant $\bar{r}$ for each $r$ in a countable $\Pi$-subalgebra $\mathcal{C}$ of $[0,1])$ and by adding the corresponding book-keeping axioms for the truth-constants. We first show that the corresponding logics $\Pi(\mathcal{C})$ are algebraizable, and hence complete with respect to the variety of $\Pi(\mathcal{C})$-algebras. The main result of the paper is the canonical standard completeness of these logics, that is, theorems of $\Pi(\mathcal{C})$ are exactly the 1-tautologies of the algebra defined over the real unit interval where the truth-constants are interpreted as their own values. It is also shown that they do not enjoy the canonical strong standard completeness, but they enjoy it for finite theories when restricted to evaluated $\Pi$-formulas of the kind $\bar{r} \rightarrow \varphi$, where $\bar{r}$ is a truth-constant and $\varphi$ a formula not containing truth-constants. Finally we consider the logics $\Pi_{\Delta}(\mathcal{C})$, the expansion of $\Pi(\mathcal{C})$ with the well-known Baaz's projection connective $\Delta$, and we show canonical finite strong standard completeness for them.
\end{abstract}

Keywords: non-classical logic, fuzzy logic, Product Logic, truth-constants, standard completeness

\section{Introduction}

Fuzzy logical systems in narrow sense are systems of mathematical many-valued logic aiming at providing a formal basis to Zadeh's fuzzy logic. The most popular are calculi with values in the real unit interval $[0,1]$ defined by a conjunction $\&$ and an implication $\rightarrow$ interpreted respectively by a left-continuous t-norm ${ }^{1} *$ and its residuum $\Rightarrow$, and where negation is defined as $\neg \varphi=\varphi \rightarrow \overline{0}$, with $\overline{0}$ being the falsity truth-constant. Among this class of systems, known

\footnotetext{
${ }^{1} \mathrm{~A}$ t-norm $*$ is a binary operation on $[0,1]$ which is commutative, associative, non decreasing in both arguments and satisfying $x * 1=x$ for all $x \in[0,1]$.
} 
as t-norm based fuzzy logics, there are three outstanding logics. Two of them were already defined before fuzzy logic was born, namely the well-known infinitely-valued Łukasiewicz Logic and Gödel Logic, also known as Dummett Logic, which turn out to correspond to the calculi defined by the so-called Łukasiewicz t-norm $x *_{\mathrm{L}} y=\max (0, x+y-1)$ and minimum t-norm $x *_{G} y=\min (x, y)$ and their residua respectively. Much later, already motivated by research on fuzzy logic, the many-valued logic $\Pi$ corresponding to the product t-norm $x *_{\Pi} y=x \cdot y$ and its residuum, called Product Logic, was axiomatized by Hájek et al. in [16, 14]. All these logics enjoy standard completeness, that is, completeness with respect to interpretations over the algebra on the unit real interval $[0,1]$ defined by the corresponding t-norm and its residuum. These three logics play a crucial role due to the fact that any continuous t-norm is an ordinal sum of isomorphic copies of Łukasiewicz, minimum and product t-norms. In [14], Hájek introduced the Basic Fuzzy Logic BL as a common fragment of the above mentioned three many-valued logics. In fact, it was an axiomatization of the tautologies common to all calculi defined by continuous t-norms.

T-norm based fuzzy logics are basically logics of comparative truth. In fact, the residuum $\Rightarrow$ of a (left-continuous) t-norm $*$ satisfies the condition $x \Rightarrow y=1$ if, and only if,$x \leq y$ for all $x, y \in[0,1]$. This means that a formula $\varphi \rightarrow \psi$ is a logical consequence of a theory if the truth degree of $\varphi$ is at most as high as the truth degree of $\psi$ in any interpretation which is a model of the theory. This is fine, but in some situations one might be also interested to explicitly represent and reason with partial degrees of truth. To do so, one convenient and elegant way is introducing truth-constants into the language. This approach actually goes back to Pavelka [24] who built a propositional many-valued logical system which turned out to be equivalent to the expansion of Eukasiewicz Logic by adding into the language a truthconstant $\bar{r}$ for each real $r \in[0,1]$, together with a number of additional axioms. Although the resulting logic is not strongly complete (like Eukasiewicz Logic), Pavelka proved that his logic, which we shall call PL, is complete in a weaker sense. Namely, by defining the truth degree of a formula $\varphi$ in a theory $T$ as

$$
\|\varphi\|_{T}=\inf \{e(\varphi) \mid e \text { evaluation model of } T\}
$$

and the provability degree of $\varphi$ in $T$ as

$$
|\varphi|_{T}=\sup \left\{r \mid T \vdash_{P L} \bar{r} \rightarrow \varphi\right\},
$$

Pavelka proved that these two degrees coincide. This kind of completeness is usually known as Pavelka-style completeness, and strongly relies on the continuity of Łukasiewicz truth functions. Novák extended Pavelka's approach to Eukasiewicz first order logic [21, 22].

Later, Hájek [14] showed that Pavelka's logic PL could be significantly simplified while keeping the completeness results. Indeed he showed it is enough to extend the language only by a countable number of truth-constants, one for each rational in $[0,1]$, and by two additional axiom schemata, called book-keeping axioms:

$$
\begin{aligned}
& \bar{r} \& \bar{s} \leftrightarrow \overline{r * s} \\
& \bar{r} \rightarrow \bar{s} \leftrightarrow \bar{r} \Rightarrow s
\end{aligned}
$$

where $*$ and $\Rightarrow$ are the Eukasiewicz t-norm and its residuum respectively. He called this new system Rational Pavelka Logic, RPL for short. Moreover, he proved that RPL is strongly complete for finite theories. 
Similar rational expansions for other continuous t-norm based fuzzy logics can be analogously defined, but Pavelka-style completeness cannot be obtained since Łukasiewicz Logic is the only fuzzy logic whose truth-functions are a continuous t-norm and a continuous residuum. ${ }^{2}$ Among different works in this direction we may cite [14] where an expansion of $G_{\Delta}$ (the expansion of Gödel Logic with Baaz's projection connective $\Delta$ ) with a finite number of rational truth-constants, and [9] where the authors define logical systems obtained by adding (rational) truth-constants to $G_{\sim}$ (Gödel Logic with an involutive negation) and to $\Pi$ (Product Logic) and $\Pi_{\sim}$ (Product Logic with an involutive negation). In the case of the rational expansions of $\Pi$ and $\Pi_{\sim}$ an infinitary inference rule (from $\{\varphi \rightarrow \bar{r}: r \in \mathbb{Q} \cap[0,1]\}$ infer $\varphi \rightarrow \overline{0}$ ) is introduced in order to get Pavelka's style completeness.

Rational truth-constants have been also considered in some stronger logics like in the logic $\mathrm{E} \frac{1}{2}$ [10], a logic that combines the connectives from both Łukasiewicz and Product logics plus the truth-constant $\overline{1 / 2}$, and in the logic PŁ [17], a logic which combines Eukasiewicz Logic connectives plus the Product Logic conjunction (but not implication), as well as in some closely related logics.

More recently, in [11] some of the authors have considered the expansion of Gödel and weak Nilpotent Minimum logics (and some of its axiomatic extensions) with rational truthconstants. Canonical standard completeness is shown for those logics as well as canonical finite strong standard completeness when restricted to formulas of the kind $\bar{r} \rightarrow \varphi$, where $\bar{r}$ denotes the truth-constant $r$ and $\varphi$ is a formula without truth-constants. Actually, this kind of formulas have been extensively considered in other frameworks for reasoning with partial degrees of truth, like in Novák's evaluated syntax formalism based on Łukasiewicz Logic (see e.g. [23]) or in fuzzy logic programming (see e.g. [25]). In particular, these formulas can be seen as a special kind of Novák's evaluated formulas, which are expressions $a / A$ where $a$ is a truth value (from a given algebra) and $A$ is a formula that may contain truth-constants again, and whose interpretation is that the truth-value of $A$ is at least $a$. Hence our formulas $\bar{r} \rightarrow \varphi$ would be expressed as $r / \varphi$ in Novák's evaluated syntax. On the other hand, formulas $\bar{r} \rightarrow \varphi$ when $\varphi$ is a Horn-like rule of the form $b_{1} \& \ldots \& b_{n} \rightarrow h$ also correspond to typical fuzzy logic programming rules $\left(b_{1} \& \ldots \& b_{n} \rightarrow h, r\right)$, where $r$ specifies a lower bound for the validity of the rule. Finally, truth-degrees in the syntax also appear in the Gerla's framework of abstract fuzzy logics [12] which is based on the notion of fuzzy consequence operators over fuzzy sets of formulas, where the membership degree of formulas are again interpreted as lower bounds of their truth degrees.

In this paper we consider general expansions à la Pavelka of the Product Logic $\Pi$ with countable subsets of truth-constants closed by the Product Logic truth-functions, and we prove canonical standard completeness for them. More specifically, after some preliminaries in the next section, in Section 3 we define the logic $\Pi(\mathcal{C})$ as the expansion of Product Logic with a countable set of truth-constants, indexed by elements of a countable $\Pi$-subalgebra $\mathcal{C}$

\footnotetext{
${ }^{2}$ An easy argument shows that for logics based on other continuous t-norms Pavelka style completeness does not hold. Let $\mathrm{L}_{*}$ be the logic of a continuous t-norm $*$ (not isomorphic to Eukasiewicz t-norm) and its residuum $\Rightarrow$ (as defined in [10]). Then it is known that the induced negation $\neg x=x \Rightarrow 0$ is not continuous in $x=0$, i.e. $\sup \{\neg x \mid x>0\}<\neg 0=1$.

Let $p$ be a propositional variable and let $T=\{p \rightarrow \bar{r} \mid r>0\}$. One can show that $\|p \rightarrow \overline{0}\|_{T} \neq|p \rightarrow \overline{0}|_{T}$. Indeed, $\|p \rightarrow \overline{0}\|_{T}=\inf \{e(p) \Rightarrow 0 \mid e(p) \leq r$ for all $r>0\}=0 \Rightarrow 0=1$, and we show that $|p \rightarrow \overline{0}|_{T}<1$. For this, it is enough to prove that $T \forall \overline{r_{0}} \rightarrow(p \rightarrow \overline{0})$ for any $r_{0}<1$ such that $r_{0}>\sup \{\neg x \mid x>0\}$ (such an element exists because $*$ is not isomorphic to Eukasiewicz t-norm). Suppose not. In such a case, there would exist a finite theory $T_{0} \subseteq T$ such that $T_{0} \vdash \overline{r_{0}} \rightarrow(p \rightarrow \overline{0})$. Then, by soundness, it should be $r_{0} \leq \neg e(p)$ for any evaluation $e$ such that $e(p) \leq s$, where $s=\min \left\{r \mid \bar{r} \rightarrow p \in T_{0}\right\}$, which is a contradiction (e.g. take $e(p)=s$ ).
} 
of $[0,1]$, and the corresponding variety of $\Pi(\mathcal{C})$-algebras. $\Pi(\mathcal{C})$-algebras are just $\Pi$-algebras having elements interpreting the truth-constants and satisfying the book-keeping equations. We show there are two types of $\Pi(\mathcal{C})$-algebras, namely type I algebras, where different truthconstants are interpreted by different elements of the algebra, and type II algebras where all constants $\bar{r}$ with $r>0$ are interpreted by the top element of the algebra. In Section 4 we study the $\Pi(\mathcal{C})$-algebras on $[0,1]$, the standard $\Pi(\mathcal{C})$-algebras, proving that the variety generated by them is indeed generated by the canonical standard $\Pi(\mathcal{C})$-algebra, $[0,1]_{\Pi(\mathcal{C})}$, i.e the standard $\Pi(\mathcal{C})$-algebra where the truth-constants are interpreted as their own values. This is used in Section 5 to prove that the $\operatorname{logic} \Pi(\mathcal{C})$ has the canonical standard completeness, i.e. it is complete for theorems with respect to the canonical standard algebra $[0,1]_{\Pi(\mathcal{C})}$, and we obtain, as a side result, that any linearly ordered $\Pi(\mathcal{C})$-algebra of type $\mathrm{I}$ is partially embeddable into the canonical standard $\Pi(\mathcal{C})$-algebra. In Section 6, we study the issue of canonical finite strong standard completeness, in particular for the sublanguage of evaluated $\Pi$-formulas. Finally, in Section 7, we extend the previous results for the expansions of Product Logic augmented with the $\Delta$ projection connective. We conclude with some final remarks and open questions for future research.

\section{Preliminaries}

Our general logical framework is the so-called Product Logic $\Pi$ defined in [16] as a propositional logic in the language $\mathcal{L}=\{\&, \rightarrow, \overline{0}\}$. Other usual connectives are definable, in particular $\overline{1}$ is $\varphi \rightarrow \varphi, \neg \varphi$ is $\varphi \rightarrow \overline{0}, \varphi \wedge \psi$ is $(\varphi \&(\varphi \rightarrow \psi)), \varphi \vee \psi$ is $((\varphi \rightarrow \psi) \rightarrow \psi) \wedge((\psi \rightarrow \varphi) \rightarrow \varphi)$, and $\varphi \equiv \psi$ is $(\varphi \rightarrow \psi) \wedge(\psi \rightarrow \varphi)$. We will denote by $F m_{\mathcal{L}}$ the set of well-formed formulas built over the language $\mathcal{L}$ and a countable set of propositional variables $V a r$. Axioms of $\Pi$ are ${ }^{3}$ :

The rule of inference of $\Pi$ is modus ponens.

We note that (A1) - (A7) defines, together with modus ponens, Hájek's Basic Fuzzy Logic $B L$ [14]. This emphasizes that $\Pi$ is in fact the extension of $B L$ with axioms (П1) and (П2). Moreover, adding to $B L$ the axioms $\varphi \rightarrow \varphi \& \varphi$ or $\neg \neg \varphi \rightarrow \varphi$ we obtain Gödel Logic and Łukasiewicz Logic respectively.

The (finitary) notion of proof in $\Pi$ is as usual from the above axioms and inference rule. If $T$ is an arbitrary theory we shall write $T \vdash_{\Pi} \varphi$ to denote that there exists a proof of $\varphi$ from $T$.

\footnotetext{
${ }^{3}$ These are the original axioms given in $[16,14]$. Later Cintula has given in [6] a simpler axiomatization

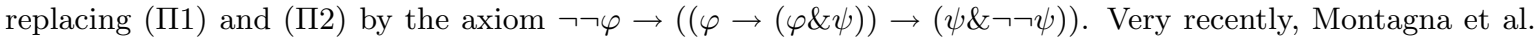
[20] have also provided this alternative axiom $\neg \varphi \vee((\varphi \rightarrow(\varphi \& \psi)) \rightarrow \psi)$ to replace (П1) and (П2).
} 
The algebraic semantics for the Product Logic $\Pi$ is given by the variety of Product algebras, in short $\Pi$-algebras, which is a subvariety of the variety of $B L$-algebras (cf. [14]), which provides an algebraic semantics for BL logic. A $B L$-algebra $\mathcal{A}=(A, \odot, \Rightarrow, \wedge, \vee, 0,1)$ is a bounded commutative pre-linear integral residuated lattice satisfying the divisibility condition

$$
x \wedge y=x \odot(x \Rightarrow y)
$$

for all $x, y \in A$. Then a $\Pi$-algebra is a just a BL-algebra such that for all $x, y, z \in A$ :

- $x \wedge \neg x=0$

- if $x \neq 0$ then $x \odot y=x \odot z$ implies $y=z$

The only finite non-trivial chain (i.e. linearly ordered algebra) in the variety of $\Pi$ algebras is the two element Boolean algebra. The so-called canonical standard П-algebra is the algebra on the unit real interval defined by the standard product and its residuum, $[0,1]_{\Pi}=\left([0,1], \min , \max , \cdot, \Rightarrow_{\Pi}, 0,1\right)$, where $\cdot$ denotes the standard product of real numbers and $x \Rightarrow_{\Pi} y=1$ if $x \leq y$ and $x \Rightarrow_{\Pi} y=y / x$ otherwise.

Product algebras are closely related to ordered Abelian groups [14], in fact a linearly ordered $\Pi$-algebra without the 0 can be identified with the negative cone of a linearly ordered Abelian group. Namely, given a linearly ordered Abelian group $\mathcal{G}=(G, \otimes, 1, \leq)$, let $\operatorname{Cone}^{-}(\mathcal{G})=\{x \in G \mid x \leq 1\}$ and define the structure $\mathbf{P}(\mathcal{G})=\left(\right.$ Cone $\left.^{-}(\mathcal{G}) \cup\{0\}, \otimes^{\prime}, \Rightarrow_{\otimes}, \min , \max , 0,1\right)$ where 0 is a new element and the operations are defined as follows:

$$
\begin{aligned}
& x \otimes^{\prime} y=x \otimes y, \text { for } x, y \in \text { Cone }^{-}(\mathcal{G}) \\
& 0 \otimes^{\prime} x=x \otimes^{\prime} 0=0, \text { for all } x \in \text { Cone }^{-}(\mathcal{G}) \cup\{0\} \\
& x \Rightarrow \otimes y= \begin{cases}1, & \text { if either } x=0 \text { or } x \leq y \text { for } x, y \in \text { Cone }^{-}(\mathcal{G}) \\
y \otimes x^{-1}, & \text { if } x>y \text { for } x, y \in \text { Cone }^{-}(\mathcal{G}) \\
0, & \text { if } x \in \text { Cone }^{-}(\mathcal{G}) \text { and } y=0\end{cases}
\end{aligned}
$$

where $x^{-1}$ denotes the inverse of $x$ with respect to the group operation $\otimes$. Then $\mathbf{P}(\mathcal{G})$ is a product algebra. And conversely, given a linearly ordered $\Pi$ - algebra $\mathcal{A}$, there is a linearly ordered group $\mathcal{G}$ such that $\mathcal{A}=\mathbf{P}(\mathcal{G})$.

Given a $\Pi$ - algebra $\mathcal{A}=(A, \odot, \Rightarrow, \wedge, \vee, 0,1)$ an $\mathcal{A}$-evaluation $e$ is a mapping $e: \operatorname{Var} \rightarrow A$ which extends to arbitrary formulas by means of the algebra operations:

$$
\begin{aligned}
& e(\overline{0})=0 \\
& e(\varphi \& \psi)=e(\varphi) \odot e(\psi) \\
& e(\varphi \rightarrow \psi)=e(\varphi) \Rightarrow e(\psi)
\end{aligned}
$$

An $\mathcal{A}$-evaluation $e$ is an $\mathcal{A}$-model of a formula $\varphi$ if $e(\varphi)=1$. $\varphi$ is an $\mathcal{A}$-tautology if $e(\varphi)=1$ for all $\mathcal{A}$-evaluations $e$. If $T$ is a theory, we write $T=_{\mathcal{A}} \varphi$ when $e(\varphi)=1$ for all $\mathcal{A}$-evaluations $e$ which are a model of all formulas in $T$.

Completeness results for $\Pi$ logic $[16,14]$ read as follows. For any finite theory $T$ and formula $\varphi$ the following conditions are equivalent:

(i) $T \vdash \Pi \varphi$ 
(ii) $T=_{\mathcal{A}} \varphi$ for each $\Pi$-algebra $\mathcal{A}$

(iii) $T \models_{\mathcal{A}} \varphi$ for each linearly ordered $\Pi$-algebra $\mathcal{A}$

(iv) $T \models[0,1]_{\Pi} \varphi$

Actually the equivalence between (i), (ii) and (iii) also holds true for arbitrary theories.

\section{Expanding Product Logic with truth-constants}

Let $C$ be a countable subset of $[0,1]$ such that $\mathcal{C}=\left(C, \cdot, \Rightarrow_{\Pi}, \min , \max , 0,1\right)$ is a (product) subalgebra of $[0,1]_{\Pi}$. Relevant examples of sets $C$ are:

(i) the set $[0,1]_{\mathbb{Q}}=\mathbb{Q} \cap[0,1]$ of rational numbers in $[0,1]$,

(ii) the sets $N P\left[a_{1}, \ldots, a_{m}\right]=\left\{a_{1}^{z_{1}} \cdot \ldots \cdot a_{m}^{z_{m}} \mid z_{1}, \ldots, z_{m} \in \mathbb{Z}, a_{1}^{z_{1}} \cdot \ldots \cdot a_{m}^{z_{m}}<1\right\} \cup\{0,1\}$, for any reals $0<a_{1}<\ldots<a_{m}<1$,

(iii) the sets $R P\left[a_{1}, \ldots, a_{m}\right]=\left\{a_{1}^{r_{1}} \cdot \ldots \cdot a_{m}^{r_{m}} \mid r_{1}, \ldots, r_{m} \in \mathbb{Q}, a_{1}^{r_{1}} \cdot \ldots \cdot a_{m}^{r_{m}}<1\right\} \cup\{0,1\}$ for any reals $0<a_{1}<\ldots<a_{m}<1$,

Definition 3.1. Given such a $\mathcal{C}$, consider a countable set $\bar{C}=\{\bar{c} \mid c \in C\}$ of truth-constants and the expanded language $\mathcal{L}_{C}=\mathcal{L} \cup \bar{C}$. The expansion of Product logic with truth-constants from the algebra $\mathcal{C}$ is denoted as $\Pi(\mathcal{C})$ and it is the logic defined by the Hilbert-style calculus in the language $\mathcal{L}_{C}$ whose axioms are the axioms of Product Logic $\Pi$ plus the following bookkeeping axioms:

$$
\begin{aligned}
& \bar{r} \& \bar{s} \equiv \overline{r \cdot s} \\
& \bar{r} \rightarrow \bar{s} \equiv \bar{r} \bar{\Pi}_{\Pi}
\end{aligned}
$$

for all $r, s \in C$, and the only inference rule is Modus Ponens. We will use the notation $\vdash_{\Pi(\mathcal{C})}$ to refer to proofs in $\Pi(\mathcal{C})$.

Like in Product Logic, the following form of the local deduction theorem holds for $\Pi(\mathcal{C})$ :

$$
\Gamma, \psi \vdash_{\Pi(\mathcal{C})} \varphi \text { iff there exists } n \text { such that } \Gamma \vdash_{\Pi(\mathcal{C})} \psi^{n} \rightarrow \varphi
$$

for any set $\Gamma$ of $\Pi(\mathcal{C})$-formulas and $\Pi(\mathcal{C})$-formulas $\psi$ and $\varphi$. As usual $\psi^{n}$ is an abbreviation of $\psi \& \stackrel{n}{n} \& \psi$.

Definition 3.2. $A \Pi(\mathcal{C})$-algebra is a structure $\mathcal{A}=\left(A, \odot, \Rightarrow, \wedge, \vee,\left\{\bar{r}^{A}\right\}_{r \in C}\right)$, where $\mathcal{A}=$ $(A, \odot, \Rightarrow$,

$\left.\wedge, \vee, \overline{0}^{A}, \overline{1}^{A}\right)$ is a $\Pi$-algebra, and the following book-keeping equations hold for any $r, s \in C$ :

$$
\begin{aligned}
& \bar{r}^{A} \odot \bar{s}^{A}=\overline{r \cdot s}^{A} \\
& \bar{r}^{A} \Rightarrow \bar{s}^{A}=\overline{r \Rightarrow \Pi s^{A}}
\end{aligned}
$$

Given a $\Pi(\mathcal{C})$-algebra $\mathcal{A}$, an $\mathcal{A}$-evaluation $e$ is just an $\mathcal{A}$-evaluation which is extended by $e(\bar{r})=\bar{r}^{A}$ for all $r \in C$. The notions of $\mathcal{A}$-model, $\mathcal{A}$-tautology and logical consequence $\models_{\mathcal{A}}$ are then as in the case of $\Pi$ logic.

One can check that the logic $\Pi(\mathcal{C})$ is algebraizable (cf. [11]) and its equivalent algebraic semantics is given by the variety of $\Pi(\mathcal{C})$-algebras. As a consequence of this we have the following result. 
Theorem 3.3 (General Completeness). Let $T$ be an arbitrary theory over $\Pi(\mathcal{C})$ and let $\varphi$ be a formula of $\Pi(\mathcal{C})$. Then the following conditions are equivalent:

- $T \vdash_{\Pi(\mathcal{C})} \varphi$

- $T \models_{\mathcal{A}} \varphi$ for all $\Pi(\mathcal{C})$-algebras $\mathcal{A}$

- $T=_{\mathcal{A}} \varphi$ for all linearly ordered $\Pi(\mathcal{C})$-algebras $\mathcal{A}$

From this result, and using the standard completeness of Product Logic and the above mentioned local deduction theorem for $\Pi(\mathcal{C})$, one can show that the $\Pi(\mathcal{C})$ logics are conservative expansions of $\Pi$ (similarly to [11, Prop. 9] in the context of expansions of any axiomatic extension of MTL with rational truth-constants).

Corollary 3.4. $\Pi(\mathcal{C})$ is a conservative expansion of $\Pi$, i.e. if $\Gamma \cup\{\varphi\}$ is a set of $\Pi$-formulas, then $\Gamma \vdash_{\Pi(\mathcal{C})} \varphi$ iff $\Gamma \vdash_{\Pi} \varphi$.

The main aim of this paper is to refine the above general completeness when $T=\emptyset$ showing that we can restrict ourselves not only to linearly ordered algebras but to a single linearly ordered $\Pi(\mathcal{C})$-algebra, the so-called canonical standard $\Pi(\mathcal{C})$-algebra. The canonical standard $\Pi(\mathcal{C})$-algebra is the algebra $[0,1]_{\Pi(\mathcal{C})}$ over the standard Product algebra interval $[0,1]_{\Pi}$ where the truth-constants are interpreted as their own values, i.e.

$$
[0,1]_{\Pi(\mathcal{C})}=\left([0,1], \cdot, \Rightarrow_{\Pi}, \min , \max ,\{r\}_{r \in C}\right)
$$

The following are some general results about the structure of linearly ordered $\Pi(\mathcal{C})$ algebras.

Lemma 3.5. Let $\mathcal{A}=\left(A, \odot, \Rightarrow, \wedge, \vee,\left\{\bar{r}^{A}\right\}_{r \in C}\right)$ be a $\Pi(\mathcal{C})$-chain. Then:

(i) Either $\bar{r}^{A}<\bar{s}^{A}$ for any $r, s \in C$ such that $r<s$, or $\bar{r}^{A}=\overline{1}^{A}$ for all $r>0$.

(ii) $A$ is finite iff $A=\left\{\overline{0}^{A}, \overline{1}^{A}\right\}$.

Proof. Assume $r, s \in C$ are such that $0<r<s$ and $\bar{r}^{A}=\bar{s}^{A}$. Since $r<s, r / s=s \Rightarrow_{\Pi} r<1$. Hence, for any $t \in(0,1)$, there exists $n$ such that $\left(s \Rightarrow_{\Pi} r\right)^{n}<t$. By the book-keeping axioms we have ${\overline{\left(s \Rightarrow_{\Pi} r\right)^{n}}}^{A} \leq \bar{t}^{A}$. But ${\overline{\left(s \Rightarrow_{\Pi} r\right)^{n}}}^{A}=\left(\bar{s}^{A} \Rightarrow \bar{r}^{A}\right)^{n}=\overline{1}^{A}$, hence $\bar{t}^{A}=\overline{1}^{A}$. (ii) follows from the fact that the only finite non-trivial $\Pi$-algebra is the two-element Boolean algebra, and it can be expanded to a $\Pi(\mathcal{C})$-chain by interpreting the truth-constants in the second way described in $(i)$.

Definition 3.6. Let $C$ be infinite and let $\mathcal{A}$ be a linearly ordered $\Pi(\mathcal{C})$-algebra. We say $\mathcal{A}$ is of type I if $\bar{r}^{A} \neq \bar{s}^{A}$ for any $r \neq s$. Otherwise, if $\bar{r}^{A}=\overline{1}^{A}$ for all $r>0$, we say $\mathcal{A}$ is of type II.

In the following section we study the $\Pi(\mathcal{C})$-algebras on the real unit interval $[0,1]$. These results will be used in Section 5 to prove canonical standard completeness of $\Pi(\mathcal{C})$ logics. 


\section{$4 \Pi(\mathcal{C})$-algebras on $[0,1]$}

The canonical standard $\Pi(\mathcal{C})$-algebra $[0,1]_{\Pi(\mathcal{C})}$ is of type I. The (unique) standard $\Pi(\mathcal{C})$ algebra of type II will be denoted as $[0,1]_{\Pi(\mathcal{C})}^{*}$.

The next result is related to the so-called Hion's Lemma (see e.g. [13, Lemma 4.1.6]).

Lemma 4.1. Let $C$ be a subset of $[0,1]$ containing 0 and 1 and closed under the product of real numbers. Let $g: C \rightarrow[0,1]$ satisfy $g(x \cdot y)=g(x) \cdot g(y)$ for all $x, y \in C$ and $g(x)<g(y)$ for all $x, y \in C$ such that $x<y$. Then there exists $\alpha \in \mathbb{R}^{+}$such that $g(r)=r^{\alpha}$ for all $r \in C$.

Proof. By the assumptions on $g$, we have for all $r, s \in C, r, s>0$ and for all $i, j \in \mathbb{N}$ :

(i) if $r^{i} \leq s^{j}$ then $g(r)^{i} \leq g(s)^{j}$

(ii) if $r^{i} \geq s^{j}$ then $g(r)^{i} \geq g(s)^{j}$

Using logarithms in statements (i) and (ii) we get the following equivalent statements for all $i, j \in \mathbb{N}$ :

(i') if $i \cdot \ln r-j \cdot \ln s \leq 0$ then $i \cdot \ln g(r)-j \cdot \ln g(s) \leq 0$

(ii') if $i \cdot \ln r-j \cdot \ln s \geq 0$ then $i \cdot \ln g(r)-j \cdot \ln g(s) \geq 0$

or equivalently,

(i") if $\frac{i}{j} \geq \frac{\ln s}{\ln r}$ then $\frac{i}{j} \geq \frac{\ln g(s)}{\ln g(r)}$

(ii") if $\frac{i}{j} \leq \frac{\ln s}{\ln r}$ then $\frac{i}{j} \leq \frac{\ln g(s)}{\ln g(r)}$

The fact that these inequalities hold for all natural numbers $i, j$ implies that

$$
\frac{\ln s}{\ln r}=\frac{\ln g(s)}{\ln g(r)}
$$

Indeed, if $\frac{\ln s}{\ln r}>\frac{\ln g(s)}{\ln g(r)}$, then there is a rational number $\frac{i}{j}$ such that $\frac{\ln s}{\ln r}>\frac{i}{j}>\frac{\ln g(s)}{\ln g(r)}$. This contradicts (ii"). Similarly, $\frac{\ln s}{\ln r}<\frac{\ln g(s)}{\ln g(r)}$ contradicts (i").

Finally, taking an arbitrary strictly positive $r \in C$ and letting $\alpha=\ln g(r) / \ln r$, the above equality leads to

$$
g(s)=s^{\alpha}
$$

for each $s \in C$. This ends the proof.

Theorem 4.2. Let $\mathcal{A}$ be a $\Pi(\mathcal{C})$-algebra over $[0,1]_{\Pi}$ of type $I$. Then $\mathcal{A}$ is isomorphic to the canonical standard algebra $[0,1]_{\Pi(\mathcal{C})}$, i.e. the canonical standard $\Pi(\mathcal{C})$-algebra over $[0,1]$ is the only standard $\Pi(\mathcal{C})$-algebra of type I up to isomorphism.

Proof. Each $\Pi(\mathcal{C})$-algebra $\mathcal{A}$ over $[0,1]_{\Pi}$ of type I defines an embedding $g: C \rightarrow[0,1]$ by putting $g(r)=\bar{r}^{\mathcal{A}}$ which, due to the book-keeping axioms, is obviously a morphism with respect to product: $g(r \cdot s)=\bar{r} \cdot s^{\mathcal{A}}=\bar{r}^{\mathcal{A}} \cdot \bar{s}^{\mathcal{A}}=g(r) \cdot g(s)$. Moreover, since $\mathcal{A}$ is of type I, by Lemma 3.5 we have $g(r)<g(s)$ for every $r<s$ in $C$. By Lemma 4.1, there exists a positive 
real $\beta$ such that $\bar{r}^{\mathcal{A}}=r^{\beta}$ for each $r \in C$. Therefore, the mapping $h:[0,1] \rightarrow[0,1]$ defined as $h(x)=x^{1 / \beta}$ defines an isomorphism from $\mathcal{A}$ to $[0,1]_{\Pi(\mathcal{C})}$.

From the above results it is already clear that the variety generated by the $\Pi(\mathcal{C})$-algebras on $[0,1]$ is the variety $\mathbb{V}\left([0,1]_{\Pi(\mathcal{C})},[0,1]_{\Pi(\mathcal{C})}^{*}\right)$ generated by the algebras $[0,1]_{\Pi(\mathcal{C})}$ and $[0,1]_{\Pi(\mathcal{C})}^{*}$. In the rest of this section, we prove that this variety is in fact equal to $\mathbb{V}\left([0,1]_{\Pi(\mathcal{C})}\right)$. This will be achieved by proving that $[0,1]_{\Pi(\mathcal{C})}^{*}$ belongs to the variety generated by $[0,1]_{\Pi(\mathcal{C})}$. In order to prove this in Theorem 4.5, we need a method to convert a nonsatisfying evaluation $e$ of a $\Pi(\mathcal{C})$-formula in $[0,1]_{\Pi(\mathcal{C})}^{*}$ to a nonsatisfying evaluation $e^{\prime}$ of the same formula in $[0,1]_{\Pi(\mathcal{C})}$. This is achieved in the following paragraphs concluded by the specific result in Proposition 4.4 .

Let $e$ be an evaluation of $\Pi(\mathcal{C})$-formulas on the type II algebra $[0,1]_{\Pi(\mathcal{C})}^{*}$. In particular, for every $r \in C \backslash\{0\}$, we have $e(\bar{r})=1$. Consider the following set of evaluations $e_{t}^{\prime}$ on the canonical standard algebra $[0,1]_{\Pi(\mathcal{C})}$, parametrized by positive real numbers $t \in \mathbb{R}^{+}$, defined as follows.

- $e_{t}^{\prime}(\bar{r})=r$ for every truth-constant symbol $\bar{r}$,

- $e_{t}^{\prime}(x)=(e(x))^{t}$ for every propositional variable $x$,

- composite formulas are evaluated according to the operations in $[0,1]_{\Pi(\mathcal{C})}$.

We are going to prove that if $e(\phi)<1$, then $e_{t}^{\prime}(\phi)<1$ for every $t$ large enough. We start by making the following remarks.

The set $[0,1]^{\mathbb{R}^{+}}$of all functions from $\mathbb{R}^{+}$into $[0,1]$ becomes a $\Pi$-algebra with the operations - and $\Rightarrow_{\Pi}$ defined pointwise and with the constant function 0 as bottom and the constant function 1 as top.

Let $F \subseteq[0,1]^{\mathbb{R}^{+}}$be the set of all functions $f: \mathbb{R}^{+} \rightarrow[0,1]$ satisfying the following condition:

(E) There are $0<c \leq 1$ and $t_{0}>0$ such that $c \leq f(t)$ for all $t \geq t_{0}$.

It is immediate to verify that $F$ is an implicative filter of the $\Pi$-algebra $[0,1]^{\mathbb{R}^{+}}$(see e.g. $[4$, Lemma 1.5]). Hence the congruence relation defined by $F$ on $[0,1]^{\mathbb{R}^{+}}, f \sim g$ iff $f \Rightarrow_{\Pi} g \in F$ and $g \Rightarrow_{\Pi} f \in F$, turns out to be defined as

$f \sim g$ iff there are $0<c, d \leq 1$ and $t_{0}>0$ such that $c \cdot f(t) \leq g(t) \leq f(t) / d$ for all $t>t_{0}$,

Indeed, if $c \leq f(t) \Rightarrow g(t)$ for $t>t_{1}$, then $c \cdot f(t) \leq g(t)$, and if $d \leq g(t) \Rightarrow f(t)$, then $d \cdot g(t) \leq f(t)$, for $t>t_{2}$. Therefore $c \cdot f(t) \leq g(t) \leq f(t) / d$, for $t>\max \left(t_{1}, t_{2}\right)$.

Lemma 4.3. The congruence relation $\sim$ satisfies:

(i) $f \sim 0$ iff there exists $t_{0}$ such that $f(t)=0$ for all $t>t_{0}$

(ii) If $f \sim g$ then $\lim _{t \rightarrow \infty} g(t)=0$ iff $\lim _{t \rightarrow \infty} f(t)=0$.

Proof. Both statements are straightforward using the above equivalence. 
Proposition 4.4. Let e and $e_{t}^{\prime}$ be defined as above. For every formula $\phi$ let $g_{\phi}(t)=(e(\phi))^{t}$ and $f_{\phi}(t)=e_{t}^{\prime}(\phi)$. Then we have $f_{\phi} \sim g_{\phi}$. In particular, if $e(\phi)<1$, then $\lim _{t \rightarrow \infty} e_{t}^{\prime}(\phi)=0$.

Proof. Let us proceed by induction on the complexity of $\phi$.

1. Constants.

$r=0 . g_{\overline{0}}(t)=e(\overline{0})^{t}=0$ and $f_{\overline{0}}(t)=e_{t}^{\prime}(0)=0$, and $0 \sim 0$.

$r>0 . g_{\bar{r}}(t)=(e(\bar{r}))^{t}=1^{t}=1$ and $f_{\bar{r}}(t)=e_{t}^{\prime}(\bar{r})=r$, and obviously $r \sim 1$.

2. Variables.

Direct consequence of the definition $\left(f_{x}(t)=g_{x}(t)\right)$.

3. $\phi=\left(\psi_{1} \& \psi_{2}\right)$.

$$
\begin{aligned}
& g_{\psi_{1} \& \psi_{2}}(t)=e\left(\psi_{1} \& \psi_{2}\right)^{t}=e\left(\psi_{1}\right)^{t} \cdot e\left(\psi_{2}\right)^{t}=g_{\psi_{1}}(t) \cdot g_{\psi_{2}}(t) . \\
& f_{\psi_{1} \& \psi_{2}}(t)=e_{t}^{\prime}\left(\psi_{1} \& \psi_{2}\right)=e_{t}^{\prime}\left(\psi_{1}\right) \cdot e_{t}^{\prime}\left(\psi_{2}\right)=f_{\psi_{1}}(t) \cdot f_{\psi_{2}}(t) .
\end{aligned}
$$

Since $\sim$ is a congruence, if we suppose that $f_{\psi_{1}} \sim g_{\psi_{1}}$ and $f_{\psi_{2}} \sim g_{\psi_{2}}$, we can conclude that $f_{\psi_{1} \& \psi_{2}} \sim g_{\psi_{1} \& \psi_{2}}$.

4. $\phi=\left(\psi_{1} \rightarrow \psi_{2}\right)$.

$$
\begin{aligned}
& g_{\psi_{1} \rightarrow \psi_{2}}(t)=e\left(\psi_{1} \rightarrow \psi_{2}\right)^{t}=\left(e\left(\psi_{1}\right) \Rightarrow e\left(\psi_{2}\right)\right)^{t}=e\left(\psi_{1}\right)^{t} \Rightarrow e\left(\psi_{2}\right)^{t}=g_{\psi_{1}}(t) \Rightarrow g_{\psi_{2}}(t) . \\
& f_{\psi_{1} \rightarrow \psi_{2}}(t)=e_{t}^{\prime}\left(\psi_{1} \rightarrow \psi_{2}\right)=e_{t}^{\prime}\left(\psi_{1}\right) \Rightarrow e_{t}^{\prime}\left(\psi_{2}\right)=f_{\psi_{1}}(t) \Rightarrow f_{\psi_{2}}(t) .
\end{aligned}
$$

Using again the fact that $\sim$ is a congruence, from the hypothesis $f_{\psi_{1}} \sim g_{\psi_{1}}$ and $f_{\psi_{2}} \sim$ $g_{\psi_{2}}$, we obtain $f_{\psi_{1} \rightarrow \psi_{2}} \sim g_{\psi_{1} \rightarrow \psi_{2}}$.

The first statement of the proposition is proved. The second statement follows from the first statement and (ii) of Lemma 4.3.

Theorem 4.5. $[0,1]_{\Pi(\mathcal{C})}^{*} \in \mathbb{V}\left([0,1]_{\Pi(\mathcal{C})}\right)$, hence the variety generated by the class of $\Pi(\mathcal{C})$ algebras over the unit real interval $[0,1]$ is $\mathbb{V}\left([0,1]_{\Pi(\mathcal{C})}\right)$.

Proof. Let $\varphi$ be not valid in $[0,1]_{\Pi(\mathcal{C})}^{*}$. There exists an evaluation $e$ on $[0,1]_{\Pi(\mathcal{C})}^{*}$ such that $e(\varphi)<1$. By the above proposition, $\lim _{t \rightarrow \infty} e_{t}^{\prime}(\varphi)=0$ as well, hence for every large enough $t, e_{t}^{\prime}(\varphi)<1$. Since $e_{t}^{\prime}$ is an evaluation on $[0,1]_{\Pi(\mathcal{C})}, \varphi$ is not valid in $[0,1]_{\Pi(\mathcal{C})}$.

\section{$5 \quad$ Standard completeness results}

We recall the assumption that in what follows $\mathcal{C}$ stands for an arbitrary countable product subalgebra of $[0,1]_{\Pi}$ such that $C \neq\{0,1\}$, including the case $C=[0,1]_{\mathbb{Q}}$.

We prove canonical standard completeness of $\Pi(\mathcal{C})$ in Theorem 5.4 (i. e. we prove that the theorems of $\Pi(\mathcal{C})$ coincide with the tautologies of the canonical standard algebra $\left.[0,1]_{\Pi(\mathcal{C})}\right)$ using a result on partial embeddability (Theorem 5.3) for type I linearly ordered $\Pi(\mathcal{C})$-algebras into the canonical standard one. Theorem 5.3 extends the known partial embeddability results 
for MV and Product algebras based on the Gurevich-Kokorin theorem for Abelian ordered groups (cf [14]).

To show that the logic $\Pi(\mathcal{C})$ has the canonical standard completeness we need, due to Theorem 3.3, to show the following. For every $\Pi(\mathcal{C})$-formula $\phi$, if there exists a linearly ordered $\Pi(\mathcal{C})$-algebra $\mathcal{A}$ and an $\mathcal{A}$-evaluation $e$ such that $e(\phi)<\overline{1}^{\mathcal{A}}$, we can find an evaluation $e^{\prime}$ on the canonical standard $\Pi(\mathcal{C})$-algebra such that $e^{\prime}(\phi)<1$. In order to achieve this, we need to embed the values of all subformulas of $\phi$ into $[0,1]$ in such a way that the existing products and residues are preserved. We will do it first for a general finite set of values in $A$.

Let $E$ be a finite subset of $A$. Denote by $C_{E}$ the set $\left\{r \in C \mid \bar{r}^{A} \in E\right\}$. Let $\widetilde{\mathcal{C}_{E}}$ be the $\Pi$-algebra generated by $C_{E}$. Note that the $\Pi$-algebra generated by $E$ is naturally a $\Pi\left(\widetilde{\mathcal{C}_{E}}\right)$-algebra. Let ${\widetilde{C_{E}}}^{*}$ be $\widetilde{C_{E}}$ without 0 .

In the proof of the next proposition we make use of the ordered Abelian group $\left(\mathcal{R}^{+}\right)_{\text {lex }}^{k}$ obtained as the lexicographic product of $k$ copies of the multiplicative groups of positive reals. Its definition and some results on ordered Abelian groups are gathered in the Appendix.

Proposition 5.1. Let $\mathcal{A}$ be a linearly ordered $\Pi(\mathcal{C})$-algebra of type $I$ and let $E$ be a finite subset of $\mathcal{A}$. Let $\mathcal{A}_{E}$ be the linearly ordered $\Pi\left(\widetilde{\mathcal{C}_{E}}\right)$-algebra generated by $E$. Then $\mathcal{A}_{E}$ is isomorphic to a $\Pi\left(\widetilde{\mathcal{C}_{E}}\right)$-algebra $\mathcal{D}$ such that the following is satisfied:

- $\mathcal{D}=\mathbf{P}(\mathcal{G})$ with $\mathcal{G}$ being a subgroup of $\left(\mathcal{R}^{+}\right)_{\text {lex }}^{k}$, where $k$ is an integer.

- there is an integer $l$ and a real number $\alpha>0$ such that for every $r \in{\widetilde{C_{E}}}^{*}$, we have $\bar{r}^{\mathcal{D}}=\omega_{k, l}\left(r^{\alpha}\right)$,

where, for any $x \in(0,1]$ and natural $1 \leq l \leq k, \omega_{k, l}(x)=(1, \ldots, 1, x, 1, \ldots, 1) \in\left(\mathbb{R}^{+}\right)^{k}$ with $x$ being at coordinate with index $l$.

Proof. Taking $\mathcal{A}_{E}$ as a $\Pi$-algebra, there is a linearly ordered Abelian group $\mathcal{G}^{\prime}$ such that $\mathcal{A}_{E}=\mathbf{P}\left(\mathcal{G}^{\prime}\right)$, i.e. $\mathcal{A}_{E} \backslash\{0\}$ is the negative cone of a linearly ordered group $\mathcal{G}^{\prime}$. Since $\mathcal{A}_{E}$ is finitely generated, so is $\mathcal{G}^{\prime}$. Hence, applying Theorem 8.1 there is a natural $k$ such that $\mathcal{G}^{\prime}$ is isomorphic to a subgroup $\mathcal{G}$ of $\left(\mathcal{R}^{+}\right)_{\text {lex }}^{k}$ (see the Appendix for the definition of $\left(\mathcal{R}^{+}\right)_{\text {lex }}^{k}$ and the result).

Then $\mathcal{A}_{E}$ is also isomorphic (through a mapping $\iota$ ) to $\mathbf{P}(\mathcal{G})$ as $\Pi$-algebras. For every $r \in$ $\widetilde{C_{E}}$ define $\bar{r}^{\mathbf{P}(\mathcal{G})}=\iota\left(\bar{r}^{\mathcal{A}}\right)$. Using this, $\mathbf{P}(\mathcal{G})$ is a $\Pi\left(\widetilde{\mathcal{C}_{E}}\right)$-algebra isomorphic to $\mathcal{A}_{E}$. Therefore, for simplicity, we may assume from now that $\mathcal{A}_{E}=\mathbf{P}(\mathcal{G})$.

Since $\widetilde{\mathcal{C}_{E}}$ is an Archimedean $\Pi$-algebra, there is a unique $l \leq k$ such that for each element $\bar{r}^{\mathcal{A}}, r<1$ and $r \in{\widetilde{C_{E}}}^{*}$, we have $\bar{r}^{\mathcal{A}}=\left(1, \ldots, 1, a_{l}, \ldots, a_{k}\right)$ with $a_{l}<1$. Indeed, suppose $r, s \in$ ${\widetilde{C_{E}}}^{*}$ such that $s<r<1$ and $\bar{r}^{\mathcal{A}}=\left(1, \ldots, 1, a_{i}, \ldots, a_{k}\right)$ and $\bar{s}^{\mathcal{A}}=\left(1, \ldots, 1, b_{j}, \ldots, b_{k}\right)$ with $i>j$. There is a natural $m$ such that $r^{m}<s$, but obviously $\left(\bar{r}^{\mathcal{A}}\right)^{m}=\left(1, \ldots, 1,\left(a_{i}\right)^{m}, \ldots,\left(a_{k}\right)^{m}\right)>_{\text {lex }}$ $\left(1, \ldots, 1, b_{j}, \ldots, b_{k}\right)=\bar{s}^{\mathcal{A}}$, contradiction.

Let $f_{1}, \ldots, f_{k}:{\widetilde{C_{E}}}^{*} \rightarrow \mathbb{R}^{+}$be functions such that for each $r \in \widetilde{C_{E}}{ }^{*}$ we have $\bar{r}^{\mathcal{A}}=$ $\left(f_{1}(r), \ldots, f_{k}(r)\right)$. Due to the validity of the book-keeping axioms, for each $i, f_{i}: \widetilde{C_{E}} \rightarrow \mathbb{R}^{+}$ is a homomorphism for the product. According to the above paragraph, $f_{l}$ is the first of the functions $f_{i}$ which is not the constant 1 . Since the algebra $\mathcal{A}_{E}$ is a $\Pi\left(\widetilde{\mathcal{C}_{E}}\right)$-algebra of type I and, by the previous paragraph, $f_{l}(r)<1$ for every $r<1, r \in{\widetilde{C_{E}}}^{*}, f_{l}$ is one-to-one and preserves the order (indeed if $f_{l}(r)=f_{l}(s)$ for some $r \geq s$, then $f_{l}(r \Rightarrow s)=1$ ). Hence, by Lemma $4.1, f_{l}$ is a power and we have

$$
\bar{r}^{\mathcal{A}}=\left(1,1, \ldots, r^{\alpha}, f_{l+1}(r), \ldots, f_{k}(r)\right)
$$


for some real $\alpha>0$.

Now let $M=\left\{x_{l} \mid\left(x_{1}, \ldots, x_{l}, x_{l+1}, \ldots, x_{k}\right) \in \mathcal{G}\right\}$ be the set of all $l$-components of elements of $\mathcal{G}$. By its definition, $M$ with the multiplication is a subgroup of $\mathbb{R}^{+}$which is generated by the set of $r^{\alpha}$ 's, for $r \in{\widetilde{C_{E}}}^{*}$, and additionally by a finite number of values $x_{l}$ coming from the elements of $E$. Now, define mappings $g_{l+1}, \ldots, g_{k}: M \rightarrow \mathbb{R}^{+}$as follows:

1. put $g_{j}\left(r^{\alpha}\right)=f_{j}(r)$ for all $r \in{\widetilde{C_{E}}}^{*}$

2. using Lemma 8.2 (see Appendix), extend $g_{j}$ to the subgroup generated by $\widetilde{C_{E}}{ }^{*}$

3. finally, applying Lemma 8.3 (see Appendix) for the l-component of each element of $E$, which is not an interpretation of an element of $C_{E}$, extend $g_{j}$ to the whole $M$.

As a result, we get a homomorphism $g_{j}$ from $M$ to $\mathbb{R}^{+}$for each $j \in\{l+1, \ldots, k\}$.

Finally, define a new mapping $h: \mathcal{G} \rightarrow\left(\mathbb{R}^{+}\right)^{k}$ by putting

$$
h\left(\left(x_{1}, \ldots, x_{l}, x_{l+1}, \ldots, x_{k}\right)\right)=\left(x_{1}, \ldots, x_{l}, x_{l+1} / g_{l+1}\left(x_{l}\right), \ldots, x_{k} / g_{k}\left(x_{l}\right)\right) .
$$

We claim that, so defined, $h$ is a monomorphism. Indeed, since the $g_{j}$ 's are homomorphisms for the product on $M, h$ is a homomorphism for the product on $\mathcal{G}$ as well. If two elements of $\mathcal{G}$ differ in $x_{i}$ for $i \leq l$, then their images are ordered in the same way, since the first $l$ coordinates are not changed by $h$. If two elements of $\mathcal{G}$ agree in the first $l$ coordinates and the first different coordinate is $x_{i}$ for $i>l$, then their images are ordered in the same way, since $x_{i}$ is again the first differing coordinate and $x_{i}$ is divided by the same number in both images.

Therefore, $h(\mathcal{G})$ is a subgroup of $\left(\mathcal{R}^{+}\right)_{\text {lex }}^{k}$ which is isomorphic to $\mathcal{G}$. Consider the $\Pi$-algebra $\mathcal{D}=\mathbf{P}(h(\mathcal{G}))$. By construction of $h$, we have $h\left(\bar{r}^{A}\right)=\omega_{k, l}\left(r^{\alpha}\right)$ for every $r \in \widetilde{C_{E}}{ }^{*}$. Hence, by defining $\bar{r}^{\mathcal{D}}=h\left(\bar{r}^{A}\right)=\omega_{k, l}\left(r^{\alpha}\right)$ for every $r \in \widetilde{C_{E}}{ }^{*}, \mathcal{D}$ becomes a $\Pi\left(\widetilde{\mathcal{C}_{E}}\right)$-algebra, and moreover, $\mathcal{D}$ is isomorphic to $\mathcal{A}_{E}$. This ends the proof of Proposition 5.1.

In the following we show that there is a partial isomorphism of any $\Pi\left(\widetilde{\mathcal{C}_{E}}\right)$-algebra of the special form guaranteed by Proposition 5.1 into the canonical standard $\Pi(\mathcal{C})$-algebra.

Proposition 5.2. Let $\mathcal{G}$ be subgroup of $\left(\mathcal{R}^{+}\right)_{\text {lex }}^{k}$ such that $\mathcal{D}=\mathbf{P}(\mathcal{G})$ is a $\Pi(\mathcal{C})$-algebra, with $\bar{r}^{\mathcal{D}}=\omega_{k, l}\left(r^{\alpha}\right)$ for every $r \in C^{*}$, for some natural $l$ and positive real $\alpha$, and $\overline{0}^{\mathcal{D}}=(0, k, 0)$. Then for every finite subset $E$ of $\mathcal{D}$ there is a mapping $q: E \rightarrow[0,1]$ satisfying the following four conditions

(i) q preserves the order,

(ii) $q\left(\bar{r}^{D}\right)=r$ for all $r \in C_{E}$,

(iii) If $x, y, x * y \in E$ then $q(x) \cdot q(y)=q(x * y)$.

(iv) If $x, y, x \Rightarrow y \in E$ then $q(x) \Rightarrow_{\Pi} q(y)=q(x \Rightarrow y)$.

Proof. The candidates for $q$ are restrictions to $E$ of functions $g: \mathcal{G} \rightarrow \mathbb{R}^{+}$of the form

$$
g\left(\left(x_{1}, x_{2}, \ldots, x_{k}\right)\right)=\left(x_{1}^{\varepsilon_{1}} \cdot x_{2}^{\varepsilon_{2}} \cdot \ldots \cdot x_{k}^{\varepsilon_{k}}\right)^{\beta},
$$

where $\varepsilon_{i}, \beta>0$. Each of these functions is a homomorphism w.r.t. the product of $\mathcal{G}$. Hence, for every choice of $\varepsilon_{i}$ and $\beta$, the restriction of $g$ to $E$ satisfies (iii). By the assumption, for 
every $r \in C^{*} \bar{r}^{\mathcal{D}}=\omega_{k, l}\left(r^{\alpha}\right)$. Hence, for every choice of $\varepsilon_{i}$ and $\beta$, we have $g\left(\bar{r}^{\mathcal{D}}\right)=r^{\alpha \cdot \varepsilon_{l} \cdot \beta}$, where $\alpha \cdot \varepsilon_{l} \cdot \beta>0$. By choosing $\beta=1 /\left(\alpha \cdot \varepsilon_{l}\right)$, we obtain that the restriction of $g$ to $E$ satisfies (ii).

Let us prove that it is possible to choose the $\varepsilon_{i}$ in such a way that the restriction of $g$ to $E$ satisfies (i). Let us classify the pairs of distinct values in $E$ according to the first index $i_{0}$, where the values differ. Pairs which satisfy $i_{0}=k$ are ordered correctly for any positive value of $\varepsilon_{k}$. Pairs satisfying $i_{0}=k-1$ may be put into the right order by choosing $\varepsilon_{k-1}=1$ and $\varepsilon_{k}$ small enough to guarantee that the difference (measured as a ratio) in the $(k-1)$-th coordinate is always larger than the difference in the $k$-th coordinate. In fact, if the exponents $\varepsilon_{k-1}=1, \varepsilon_{k}$ guarantee the right order of the pairs with $i_{0}=k-1$, then the exponents $\varepsilon_{k-1}=t, t \cdot \varepsilon_{k}$, for any positive $t$, guarantee the order as well. Hence, when it is necessary to put the pairs with $i_{0}=k-2$ into the right order, we choose $\varepsilon_{k-2}=1$ and $t$ small enough so that the difference in the $(k-2)$-th coordinate is always larger than the differences contributed by $(k-1)$-th and $k$-th coordinates. Since we preserve the ratio between $\varepsilon_{k-1}$ and $\varepsilon_{k}$, we do not destroy the already correct order of pairs with $i_{0}=k-1$. We proceed in a similar way for pairs with smaller and smaller $i_{0}$.

The condition (iv), the preservation of existing implications in $E$, is a consequence of $h$ being order preserving (i) and the preservation of existing products (iii).

Theorem 5.3 (Partial embeddability for type $\mathrm{I} \Pi(\mathcal{C})$-algebras). Let $\mathcal{A}$ be a linearly ordered $\Pi(\mathcal{C})$-algebra of type $I$ and let $E$ be a finite subset of $A$. Then there exists a one-to-one mapping $h: E \rightarrow[0,1]$ satisfying the following conditions:

(i) $h$ preserves the order,

(ii) $h\left(\bar{r}^{A}\right)=r$ for all $r \in C_{E}$,

(iii) If $x, y, z \in E$ and $z=x * y$ then $h(x) \cdot h(y)=h(z)$.

(iv) If $x, y, z \in E$ and $z=x \Rightarrow y$ then $h(x) \Rightarrow_{\Pi} h(y)=h(z)$.

Proof. Let $\mathcal{D}$ be the algebra guaranteed by Proposition 5.1 applied to $\mathcal{A}_{E}$. Let $E^{\prime}$ be the image of $E$ under the isomorphism between $\mathcal{A}_{E}$ and $\mathcal{D}$. Applying Proposition 5.2 to $\mathcal{D}$ and $E^{\prime}$ with $C=\widetilde{C_{E}}$, we obtain an embedding $q$, whose composition with the above isomorphism has the required properties of $h$.

In the following, this mapping $h$ will be called a partial embedding (of the partial algebra over $E$ into $\left.[0,1]_{\Pi(\mathcal{C})}\right)$. The existence of a partial embedding for type $\mathrm{I} \Pi(\mathcal{C})$-algebras extends the known partial embeddability results for MV and Product algebras based on the GurevichKokorin theorem for Abelian ordered groups (cf [14]).

Theorem 5.4 (Canonical standard completeness). The logic $\Pi(\mathcal{C})$ has the canonical standard completeness.

Proof. Let $\varphi$ be a $\Pi(\mathcal{C})$ formula such that $\forall_{\Pi(\mathcal{C})} \varphi$. We can further assume $\varphi$ contains some truth constant $\bar{r}$ with $0<r<1$ as subformula, otherwise the standard completeness of Product Logic does the job. By general completeness, there is a linearly ordered $\Pi(\mathcal{C})$-algebra $\mathcal{A}$ and an evaluation $e$ on $\mathcal{A}$ such that $e(\varphi)<\overline{1}^{\mathcal{A}}$. The task is to find an evaluation $e^{\prime}$ on the canonical standard $\Pi(\mathcal{C})$-algebra $[0,1]_{\Pi(\mathcal{C})}$ such that $e^{\prime}(\varphi)<1$. Let $E=\{e(\psi) \mid \psi$ is a subformula of $\varphi\} \cup\left\{\overline{0}^{\mathcal{A}}, \overline{1}^{\mathcal{A}}\right\}$. We consider the following cases: 
Case 1: $\mathcal{A}$ is of type I.

By applying Theorem 5.3 we obtain a partial embedding $h$ of $E$ into [0,1]. Now define a $[0,1]_{\Pi(\mathcal{C})^{-}}$evaluation $e^{\prime}$ by putting

$$
e^{\prime}(x)= \begin{cases}h(e(p)), & \text { if } x \text { is a prop. variable in } \varphi \\ \text { arbitrary, } & \text { otherwise }\end{cases}
$$

It is easy to check then, by the properties of $h$, that $e^{\prime}(\varphi)=h(e(\varphi))<1$.

Case 2: $\mathcal{A}$ is of type II.

By the well-known results of П-algebras (see [3]), there is a partial embedding $f$ of $E$ into the standard $\Pi$-algebra $[0,1]_{\Pi}$ and the evaluation $e^{\prime}$ on $[0,1]_{\Pi}$ defined as follows

$e^{\prime}(p)= \begin{cases}f(e(p)), & \text { if } p \text { is a propositional variable in } \varphi \\ \text { arbitrary, } & \text { otherwise }\end{cases}$

is such that $e^{\prime}\left(\varphi^{*}\right)<1$, where $\varphi^{*}$ is the $\Pi$-formula obtained from $\varphi$ by replacing all truth-constants $\bar{r}$ with $0<r$ by $\overline{1}$. Now, the evaluation $e^{\prime \prime}$ on $[0,1]_{\Pi(\mathcal{C})}^{*}$, the standard $\Pi(\mathcal{C})$-algebra of type II, such that $e^{\prime \prime}(p)=e^{\prime}(p)$ for all propositional variables $p$ satisfies $e^{\prime}\left(\varphi^{*}\right)=e^{\prime \prime}(\varphi)<1$. Then, by Corollary 8 , there is also an evaluation $e^{\prime \prime \prime}$ on the canonical standard $\Pi(\mathcal{C})$-algebra $[0,1]_{\Pi(\mathcal{C})}$ such that $e^{\prime \prime \prime}(\varphi)<1$. This ends the proof of Case 2 and hence of the theorem as well.

\section{$6 \quad$ Finite Strong Completeness Results}

Since Product Logic has not the strong standard completeness for arbitrary theories this is also true for $\Pi(\mathcal{C})$, but $\Pi(\mathcal{C})$ does not even enjoy canonical strong standard completeness for finite theories. Namely, for any rational $0<r<1$ and any propositional variable $p$, $\bar{r} \nvdash_{\Pi(\mathcal{C})} p$ but it trivially holds that $\bar{r}=_{[0,1]_{\Pi(\mathcal{C})}} p$ since there is no evaluation which is a model of $\bar{r}$. Looking at this example, one could think that the reason for failure is that the theory used, $T=\{\bar{r}\}$, is somewhat special, in the sense that it is not satisfiable. Unfortunately, being satisfiable is not a sufficient condition for strong standard completeness as the following example, taken from [11], shows.

Let $T=\{\bar{r} \vee p\}$, where $0<r<1$ and $p$ is a propositional variable. It is clear that $T$ is satisfiable for any evaluation $e$ such that $e(p)=1$, and that $T \models_{[0,1]_{\Pi(\mathcal{C})}} p$. But again $T \nvdash_{\Pi(\mathcal{C})} p$ since if so, by the deduction and canonical standard completeness theorems for $\Pi(\mathcal{C})$, it should also be true that, for some $k$, then $\models_{[0,1]_{\Pi(\mathcal{C})}}(\bar{r} \vee p)^{k} \rightarrow p$, which is false for any evaluation with $e(p)<r^{k}$.

However, we can still show some interesting results. Namely, we will first show that finite strong completeness holds for the semantics obtained by considering simultaneously the $\Pi(\mathcal{C})$ algebras $[0,1]_{\Pi(\mathcal{C})}$ and $[0,1]_{\Pi(\mathcal{C})}^{*}$, but it does not hold for the semantics defined by just one of these algebras. And moreover, this result cannot be improved. Nevertheless, as usual we will continue referring to this kind of completeness as standard since any $\Pi(\mathcal{C})$-algebra over $[0,1]$ is proved to be isomorphic to one of these two . Secondly, we will show that we can indeed 
get canonical finite strong standard completeness when we restrict ourselves to formulas of the kind

$$
\bar{r} \rightarrow \varphi
$$

where $\varphi$ is a $\Pi$-formula, i.e. a formula without truth-constants (different from $\overline{0}$ and $\overline{1}$ ). We will use the name graded or evaluated $\Pi$-formulas to refer to this kind formulas. Such a formula $\bar{r} \rightarrow \varphi$ is also denoted in the literature as the pair $(\varphi, r)$.

Theorem 6.1 (Finite Strong Standard Completeness of $\Pi(\mathcal{C})$ ). For any $\Pi(\mathcal{C})$-formula $\varphi$ and any finite set of $\Pi(\mathcal{C})$-formulas $\Gamma$, we have $\Gamma \vdash_{\Pi(\mathcal{C})} \varphi$ iff $\Gamma \models_{[0,1]_{\Pi(\mathcal{C}}} \varphi$ and $\Gamma \models_{[0,1]_{\Pi(\mathcal{C}}^{*}} \varphi$.

Proof. Soundness is obvious. Suppose $\Gamma \nvdash_{\Pi(\mathcal{C})} \varphi$. Since $\Gamma$ is finite, without loss of generality we can assume it consists of a single formula (the conjunction of the formulas in $\Gamma$ ), say $\chi$. Thus we suppose $\chi \nvdash_{\Pi(\mathcal{C})} \varphi$. By general completeness, there is a linearly ordered $\Pi(\mathcal{C})$ algebra $\mathcal{A}$ and an $\mathcal{A}$-interpretation $e$ such that $e(\chi)=\overline{1}^{\mathcal{A}}$ and $e(\varphi)<\overline{1}^{\mathcal{A}}$. Take the finite set $X=\{e(\psi) \mid \psi$ subformula of $\varphi$ or $\chi\} \cup\left\{\overline{0}^{\mathcal{A}}, \overline{1}^{\mathcal{A}}\right\}$. We distinguish two cases:

(1) If $\mathcal{A}$ is of type I, i.e. if $r, s \in C$ with $r<s$, then $\bar{r}^{\mathcal{A}}<\bar{s}^{\mathcal{A}}$, then from the result of partial embeddability (Theorem 5.3) there is a partial embedding $h: X \rightarrow[0,1]$. Thus, we can define the $[0,1]_{\Pi(\mathcal{C})}$-evaluation $e^{\prime}$ by $e^{\prime}(x)=h(e(x))$ for any variable $\mathrm{x}$ appearing in $\varphi$ or $\psi$ and $e^{\prime}(x)=1$ otherwise. Obviously $e^{\prime}(\chi)=1$ and $e^{\prime}(\varphi)<1$. Hence $\chi \forall_{[0,1]_{\Pi(\mathcal{C})}} \varphi$.

(2) Suppose $\mathcal{A}$ is a $\Pi(\mathcal{C})$-algebra of type II, i.e. $\bar{r}^{A}=\overline{1}^{\mathcal{A}}$ for all $r \in C-\{0\}$. Then there is a partial embedding (as Product algebras) $h$ of $X$ into the standard $\Pi$-algebra $[0,1]_{\Pi}$, and since $h\left(\overline{1}^{A}\right)=1, h \circ e$ can be easily extended to a full $[0,1]_{\Pi(\mathcal{C})^{*}}^{*}$-evaluation $e^{\prime}$ such that $e^{\prime}(\chi)=1$ and $e^{\prime}(\varphi)<1$. Hence $\chi \forall_{[0,1]_{\Pi(\mathcal{C})}^{*}} \varphi$.

As mentioned before, it turns out that this finite strong completeness cannot be improved in the sense that it cannot be proved with respect to the semantics given by a single algebra. Namely, given $r \in C$ such that $0<r<1$, we have already seen that $p \vee \bar{r} \models_{[0,1]_{\Pi(\mathcal{C})}} p$ but $p \vee \bar{r} \not \models_{[0,1]_{\Pi(\mathcal{C})}^{*}} p$. Conversely, $p \models_{[0,1]_{\Pi(\mathcal{C})}^{*}} p \wedge \bar{r}$ but $p \nvdash_{[0,1]_{\Pi(\mathcal{C})}} p \wedge \bar{r}$.

Next we will show that we can improve the finite strong standard completeness result when we restrict ourselves to graded $\Pi$-formulas. Indeed the following canonical finite strong standard completeness result holds:

$$
\begin{gathered}
\left\{\bar{r}_{i} \rightarrow \varphi_{i} \mid i=1, . ., n\right\} \vdash_{\Pi(\mathcal{C})} \bar{s} \rightarrow \psi \\
\text { if, and only if, } \\
\left\{\bar{r}_{i} \rightarrow \varphi_{i} \mid i=1, . ., n\right\} \models_{[0,1]_{\Pi(\mathcal{C})}} \bar{s} \rightarrow \psi
\end{gathered}
$$

where $\varphi_{i}$ and $\psi$ are $\Pi$-formulas, i.e., formulas not containing truth-constants different from $\overline{0}$ and $\overline{1}$.

Actually, as always, one direction (soundness) is easy due to the book-keeping axioms. To prove the converse direction

$$
\begin{aligned}
& \text { If }\left\{\bar{r}_{i} \rightarrow \varphi_{i} \mid i=1, . ., n\right\} \models_{[0,1]_{\Pi(\mathcal{C})}} \bar{s} \rightarrow \psi \text { then } \\
& \quad\left\{\bar{r}_{i} \rightarrow \varphi_{i} \mid i=1, . ., n\right\} \vdash_{\Pi(\mathcal{C})} \bar{s} \rightarrow \psi
\end{aligned}
$$

it is enough to combine Theorem 6.1 with the following result. 
Lemma 6.2. If $\left\{\bar{r}_{i} \rightarrow \varphi_{i} \mid i=1, . ., n\right\} \models_{[0,1]_{\Pi(\mathcal{C})}} \bar{s} \rightarrow \psi$ then $\left\{\bar{r}_{1} \rightarrow \varphi_{1}, \ldots, \bar{r}_{n} \rightarrow\right.$ $\left.\varphi_{n}\right\} \models[0,1]_{\Pi(\mathcal{C})}^{*} \bar{s} \rightarrow \psi$

Proof. Without loss of generality we may assume $r_{i}>0$ for all $i$ and $s>0$. Suppose $\left\{\bar{r}_{1} \rightarrow \varphi_{1}, \ldots, \bar{r}_{n} \rightarrow \varphi_{n}\right\} \forall_{[0,1]_{\Pi(\mathcal{C})}^{*}} \bar{s} \rightarrow \psi$ Then there exists a $[0,1]_{\Pi(\mathcal{C})}^{*}$-evaluation $e$ such that $e\left(\bar{r}_{1} \rightarrow \varphi_{1}\right)=\ldots=e\left(\bar{r}_{n} \rightarrow \varphi_{n}\right)=1$ and $e(\bar{s} \rightarrow \psi)<1$. Since $e\left(r_{i}\right)=e(s)=1$ for all $i$, we also have $e\left(\varphi_{1}\right)=\ldots=e\left(\varphi_{n}\right)=1$ and $e(\psi)<1$.

Assume $e(\psi)=0$. Then, letting $e^{\prime}$ be the $[0,1]_{\Pi(\mathcal{C})}$-evaluation defined by $e^{\prime}(p)=e(p)$ for any propositional variable $p$, we have $e^{\prime}\left(\overline{r_{1}} \rightarrow \varphi_{1}\right)=\ldots=e^{\prime}\left(\overline{r_{n}} \rightarrow \varphi_{n}\right)=1$ and $e^{\prime}(\psi)=0$, hence $\left\{\bar{r}_{1} \rightarrow \varphi_{1}, \ldots, \bar{r}_{n} \rightarrow \varphi_{n}\right\} \not \forall_{[0,1]_{\Pi(\mathcal{C})}} \bar{s} \rightarrow \psi$.

Assume $e(\psi)>0$. Let $\alpha \in \mathbb{R}^{+}$such that $(e(\psi))^{\alpha}<s$. Then the $[0,1]_{\Pi(\mathcal{C})^{-e v a l u a t i o n}} e^{\prime}$, where $e^{\prime}(p)=(e(p))^{\alpha}$ for any propositional variable $p$, is such that $e^{\prime}\left(\overline{r_{i}} \rightarrow \varphi_{i}\right)=1$ for all $i$ but $e^{\prime}(\bar{s} \rightarrow \psi)<1$, hence $\left\{\bar{r}_{1} \rightarrow \varphi_{1}, \ldots, \bar{r}_{n} \rightarrow \varphi_{n}\right\} \forall_{[0,1]_{\Pi(\mathcal{C})}} \bar{s} \rightarrow \psi$.

Finally, as a direct consequence of Theorem 6.1 and the above lemma we can state the following completeness result.

Corollary 6.3 (Canonical finite strong standard completeness). For any П-formulas $\varphi_{1}, \ldots, \varphi_{n}, \psi$ and any $r_{1}, \ldots, r_{n}, s \in C$, it holds that $\left\{\bar{r}_{i} \rightarrow \varphi_{i} \mid i=1,2, . ., n\right\} \vdash_{\Pi(\mathcal{C})} \bar{s} \rightarrow \psi$ if and only if $\left\{\bar{r}_{i} \rightarrow \varphi_{i} \mid i=1,2, . ., n\right\} \models_{[0,1]_{\Pi(\mathcal{C})}} \bar{s} \rightarrow \psi$.

The result also holds if we restrict the language to formulas of type $\varphi \rightarrow \bar{r}$ such that $\varphi$ is a formula of $\Pi$, i.e. $\varphi$ does not contain a truth-constant different from $\overline{0}, \overline{1}$. In such a case the result of the corresponding Lemma 8 is valid due to the fact that for any $r>0$, it follows that $\varphi \rightarrow \bar{r}$ is trivially a tautology over $[0,1]_{\Pi(\mathcal{C})}^{*}$.

Nevertheless the result is not true if in the restricted language we allow formulas of both types as the following example shows. Namely, it is obvious that the semantical deduction

$$
(p \rightarrow q) \rightarrow \bar{r} \models q \rightarrow p
$$

is valid over the canonical standard $\Pi(\mathcal{C})$-algebra but not over $[0,1]_{\Pi(\mathcal{C})}^{*}$ if $r<1$.

To see that it is valid over the canonical standard $\Pi(\mathcal{C})$-algebra we need only to take into account that if the residuated implication function is less than 1 , the first value has to be greater than the second, i.e, for all evaluation such that $e(p \rightarrow q) \leq r<1$, then $e(q)<e(p)$.On the other hand, over $[0,1]_{\Pi(\mathcal{C})}^{*}$ the premise is always valid $(e(\bar{r})=1$ for any $r>0)$ and thus the deduction is not valid.

\section{$7 \quad$ Expanding $\Pi_{\Delta}$ with truth-constants}

A natural extension of the considered logical framework is to introduce the well-known Baaz's $\Delta$ connective into the logic. In such a case, instead of the Product Logic $\Pi$, we take as starting point the logic $\Pi_{\Delta}$, the expansion of $\Pi$ with the $\Delta$ connective as done in [14] by adding the following axiom schemata:

$(\Delta 1) \Delta \varphi \vee \neg \Delta \varphi$

$(\Delta 2) \Delta(\varphi \vee \psi) \rightarrow(\Delta \varphi \vee \Delta \psi)$ 
$(\Delta 3) \Delta \varphi \rightarrow \varphi$

$(\Delta 4) \Delta \varphi \rightarrow \Delta \Delta \varphi$

$(\Delta 5) \Delta(\varphi \rightarrow \psi) \rightarrow(\Delta \varphi \rightarrow \Delta \psi)$

and the rule of necessitation: from $\varphi$ derive $\Delta \varphi$. The algebraic semantics of $\Pi_{\Delta}$ is given by the variety of $\Pi_{\Delta}$-algebras. A $\Pi_{\Delta}$-algebra is a structure $(A, \odot, \Rightarrow, \wedge, \vee, \Delta, 0,1)$, where $(A, \odot, \Rightarrow, \wedge, \vee, 0,1)$ is a $\Pi$-algebra satisfying the following equations:

$$
\begin{aligned}
& \Delta x \vee \neg \Delta x=1 \\
& \Delta(x \vee y) \leq(\Delta x \vee \Delta y) \\
& \Delta x \rightarrow x \\
& \Delta x \leq \Delta \Delta x \\
& (\Delta x) \odot \Delta(x \rightarrow y) \rightarrow \Delta y) \\
& \Delta 1=1
\end{aligned}
$$

The standard $\Pi_{\Delta}$-algebra is the expansion of the standard Product algebra $[0,1]_{\Pi}$ with the unary operation defined by $\Delta 1=1$ and $\Delta x=0$ for all $x<1$.

The (finite strong) standard completeness of Product Logic easily extends to $\Pi_{\Delta}$ (see [14, Th. 4.1.13]). Moreover $\Pi_{\Delta}$ is also a conservative expansion of $\Pi$ (see e.g. [5]) .

As before, given a countable subset $C$ of $[0,1]$ such that $\mathcal{C}=\left(C, \cdot, \Rightarrow_{\Pi}, \min , \max , 0,1\right)$ is a (product) subalgebra of $[0,1]_{\Pi}$, we define the logic $\Pi_{\Delta}(\mathcal{C})$ as the expansion of $\Pi(\mathcal{C})$ with the $\Delta$ connective by adding the above $(\Delta 1)-(\Delta 5)$ axioms, the necessitation rule for $\Delta$ and the following book-keeping axioms

$$
\Delta \bar{r} \equiv \overline{\delta(r)}
$$

for every $r \in C$, where $\delta$ is the unary function in $[0,1]$ defined by $\delta(x)=1$ if $x=1$ and $\delta(x)=0$ otherwise. Actually, it is enough to add just one book-keeping axiom for a particular constant $0<r_{0}<1$. Indeed, for any $s \in C$ such that $0<s<1$, there exists a natural $n$ such that $s^{n} \leq r_{0}$, hence $\bar{s}^{n} \rightarrow \bar{r}_{0}$ is provable in $\Pi(\mathcal{C})$. Now, by applying the $\Delta$ axioms, the formula $(\Delta \bar{s})^{n} \rightarrow \Delta \overline{r_{0}}$ can be derived, hence, $\neg(\Delta \bar{s})^{n}$ as well, and by reasoning in $\Pi$ one finally derives $\neg \Delta \bar{s}$, which is in fact the book-keeping axiom for $s$ since $\delta(s)=0$.

As for the algebraic counterpart, a $\Pi_{\Delta}(\mathcal{C})$-algebra is a structure $\mathcal{A}=(A, \odot, \Rightarrow$ $\left., \wedge, \vee,\left\{\bar{r}^{A}\right\}_{r \in C}, \Delta\right)$, where $\left(A, \odot, \Rightarrow, \wedge, \vee,\left\{\bar{r}^{A}\right\}_{r \in C}\right)$ is a $\Pi(\mathcal{C})$-algebra and $(A, \odot, \Rightarrow$ $\left., \wedge, \vee, \overline{0}^{A}, \overline{1}^{A}, \Delta\right)$ is a $\Pi_{\Delta}$-algebra, satisfying further the corresponding book-keeping equations

$$
\Delta \bar{r}^{\mathcal{A}}=\overline{\delta(r)}^{\mathcal{A}}
$$

for each $r \in C$. It is clear then that if $\mathcal{A}$ is a linearly ordered $\Pi_{\Delta}(\mathcal{C})$-algebra, $\Delta \bar{r}=\overline{0}$ for all $1>r \in C$.

One can check again that the logic $\Pi_{\Delta}(\mathcal{C})$ is also algebraizable with equivalent algebraic semantics given by the variety of $\Pi_{\Delta}(\mathcal{C})$-algebras and that $\Pi_{\Delta}(\mathcal{C})$-algebras still decompose as subdirect product of linearly ordered ones.

Theorem 7.1 (General Completeness). Let $T$ be an arbitrary theory over $\Pi_{\Delta}(\mathcal{C})$ and let $\varphi$ be a formula of $\Pi_{\Delta}(\mathcal{C})$. Then the following conditions are equivalent: 
- $T \vdash_{\Pi_{\Delta}(\mathcal{C})} \varphi$

- $T \models_{\mathcal{A}} \varphi$ for all $\Pi_{\Delta}(\mathcal{C})$-algebra $\mathcal{A}$

- $T \models_{\mathcal{A}} \varphi$ for all linearly ordered $\Pi_{\Delta}(\mathcal{C})$-algebra $\mathcal{A}$

The canonical standard $\Pi_{\Delta}(\mathcal{C})$-algebra is the algebra $[0,1]_{\Pi_{\Delta}(\mathcal{C})}$ over the unit real interval $[0,1]$ where the truth-constants are interpreted as their own values, i.e.

$$
[0,1]_{\Pi(\mathcal{C})}=\left([0,1], \cdot, \Rightarrow_{\Pi}, \min , \max ,\{r\}_{r \in C}, \Delta\right)
$$

Contrary to $\Pi(\mathcal{C})$-chains, as the following lemma shows, truth-constants cannot collapse in $\Pi_{\Delta}(\mathcal{C})$ chains, and hence the $\Pi(\mathcal{C})$-reducts of $\Pi_{\Delta}(\mathcal{C})$ chains are always of type $\mathrm{I}$.

Lemma 7.2. Let $\mathcal{A}=\left(A, \odot, \Rightarrow, \wedge, \vee,\left\{\bar{r}^{A}\right\}_{r \in C}, \Delta\right)$ be a $\Pi_{\Delta}(\mathcal{C})$-chain. Then $\bar{r}^{A}<\bar{s}^{A}$ for any $r, s \in C$ such that $r<s$.

Proof. Let $r<s$ and let $t=s \rightarrow r$. If $\bar{r}^{A}=\bar{s}^{A}$, then $\bar{s}^{A} \Rightarrow \bar{r}^{A}=\overline{1}^{A}$, hence $\bar{t}^{A}=\overline{1}^{A}$, hence $\Delta\left(\bar{t}^{A}\right)=\overline{1}^{A}$. But, since $t<1$, this is in contradiction with the fact that $\Delta\left(\bar{t}^{A}\right)=\overline{0}^{A}$ for all $t<1$.

Theorem 7.3 (Canonical Standard Completeness). The logic $\Pi_{\Delta}(\mathcal{C})$ has the canonical standard completeness, that is, for any formula $\varphi, \vdash_{\Pi_{\Delta}(\mathcal{C})} \varphi$ if, and only if, $\models_{[0,1]_{\Pi_{\Delta}(\mathcal{C})}} \varphi$.

Proof. By a simple application of the partial embedding result for $\Pi(\mathcal{C})$-algebras, taking into account Lemma 7.2, that is, the simplification due to the fact that there are no $\Pi_{\Delta}(\mathcal{C})$-algebras of Type II.

The issue of (finite) strong completeness now is easier than with $\Pi(C)$. Indeed, recalling the form of the deduction theorem for $\Pi_{\Delta}, \psi \vdash_{\Pi_{\Delta}} \varphi$ iff $\vdash_{\Pi_{\Delta}} \Delta \psi \rightarrow \varphi$, one can easily prove the following.

Corollary 7.4 (Canonical finite strong standard Completeness). For any $\Pi_{\Delta}(\mathcal{C})$ formula $\varphi$ and any finite set of $\Pi_{\Delta}(\mathcal{C})$-formulas $\Gamma$, it holds that $\Gamma \vdash_{\Pi_{\Delta}(\mathcal{C})} \varphi$ iff $\Gamma \models_{[0,1]_{\Pi_{\Delta}(\mathcal{C})}} \varphi$.

Analogously to the $\Pi(\mathcal{C})$ logic, one can easily show that $\Pi_{\Delta}(\mathcal{C})$ is a conservative expansion of $\Pi_{\Delta}$, and hence of $\Pi$ as well. However, $\Pi_{\Delta}(\mathcal{C})$ is a conservative expansion of $\Pi(\mathcal{C})$ only at the level of theorems, not for theories.

Theorem 7.5. The following statements hold:

(i) $\Pi_{\Delta}(\mathcal{C})$ is a conservative expansion of $\Pi_{\Delta}$.

(ii) if $\varphi$ is a formula of $\Pi(\mathcal{C})$, then $\vdash_{\Pi_{\Delta}(\mathcal{C})} \varphi$ if, and only if, $\vdash_{\Pi(\mathcal{C})} \varphi$.

(iii) There exist $\varphi, \psi$ formulas of $\Pi(\mathcal{C})$ such that $\psi \vdash_{\Pi_{\Delta}(\mathcal{C})} \varphi$ and $\psi \nvdash_{\Pi(\mathcal{C})} \varphi$. 
Proof. (i) As for the non-trivial implication, let $T \cup\{\varphi\}$ be in the language of $\Pi_{\Delta}$, and assume $T \vdash_{\Pi_{\Delta}(\mathcal{C})} \varphi$. Then there exists a finite $T_{f} \subseteq T$ such that $T_{f} \vdash_{\Pi_{\Delta}(\mathcal{C})} \varphi$, hence $T_{f} \models_{\Pi_{\Delta}(\mathcal{C})} \varphi$. This means that every evaluation $e$ on $[0,1]_{\Pi_{\Delta}(\mathcal{C})}$ which is model of $T_{f}$ is also model of $\varphi$. But each evaluation on $[0,1]_{\Pi_{\Delta}}$ determines an evaluation on $[0,1]_{\Pi_{\Delta}(\mathcal{C})}$ respecting the values of formulas of $\Pi_{\Delta}$, and viceversa. Hence we also have $T_{f} \models_{\Pi_{\Delta}} \varphi$, and by completeness of $\Pi_{\Delta}$, $T_{f} \vdash_{\Pi_{\Delta}} \varphi$, thus $T \vdash_{\Pi_{\Delta}} \varphi$ as well.

(ii) Let $\varphi$ be a formula in the language of $\Pi(\mathcal{C})$ and assume $\forall_{\Pi(\mathcal{C})} \varphi$. By canonical standard completeness, there is an evaluation $e$ on the canonical standard algebra $[0,1]_{\Pi(\mathcal{C})}$ such that $e(\varphi)<1$. But this evaluation $e$ can be trivially extended to an evaluation on $[0,1]_{\Pi_{\Delta}(\mathcal{C})}$, hence $\nvdash_{\Pi_{\Delta}(\mathcal{C})} \varphi$, and thus $\nvdash_{\Pi_{\Delta}(\mathcal{C})} \varphi$.

(iii) For any $r<1$, it holds that $\bar{r} \vdash_{\Pi_{\Delta}(\mathcal{C})} \overline{0}$ but $\bar{r} \nvdash_{\Pi(\mathcal{C})} \overline{0}$.

To conclude, let us remark that strong standard completeness for $\Pi_{\Delta}(\mathcal{C})$ does not hold. In fact, this is a consequence that $\Pi_{\Delta}(\mathcal{C})$ is a conservative expansion of $\Pi$, and $\Pi$ does not enjoy strong standard completeness.

\section{Final remarks}

After the study of the expansions of Eukasiewicz Logic and Gödel Logic with truth constants in [24, 14] and in [11] respectively, in this paper we have studied the expansion with truth-constants of Product Logic, the other main fuzzy logic based on a continuous t-norm. Following [11], the approach we have used is based on the fact that the logics $\Pi(\mathcal{C})$ are algebraizable and thus we can study their associated variety of algebras. Like in the case of Product algebras, $\Pi(\mathcal{C})$-algebras are subdirect product of linearly ordered ones. Thus we have completeness results w.r.t. the class of chains of the variety. The main results of the paper are related to canonical standard completeness of the logics $\Pi(\mathcal{C})$. We have proved canonical standard completeness and some finite strong completeness results. These results are mainly based on the embeddability of any finite partial $\Pi(\mathcal{C})$-algebra of type I into the canonical standard $\Pi(\mathcal{C})$-algebra $[0,1]_{\Pi(\mathcal{C})}$, generalization to $\Pi(\mathcal{C})$-algebras of the well-known result for $\Pi$-chains. Moreover we have proved canonical finite strong standard completeness when we restrict ourselves to formulas of type $\bar{r} \rightarrow \varphi$ where $\varphi$ is a formula of Product Logic, i.e. without additional truth-constants. In addition, we have considered the expansions of those logics with Baaz's $\Delta$ connective, called $\Pi_{\Delta}(\mathcal{C})$, showing that the resulting logic has the canonical finite strong standard completeness. Actually, this result could be also easily proved in an analogous way for the corresponding expansions of Łukasiewicz and Gödel logics with truth-constants and $\Delta$.

As suggested by one referee, an alternative approach to proving canonical standard completeness for $\Pi(\mathcal{C})$ could be to add truth constants to the analytic hypersequent calculus $G \Pi$ for Product Logic defined in [19], namely, defining $G \Pi(\mathcal{C})$ by adding extra rules to $G \Pi$ to deal with truth-constants and show that $\varphi$ is derivable in $G \Pi(\mathcal{C})$ iff $\varphi$ is valid in the canonical standard $\Pi(\mathcal{C})$-algebra. The result would then follow by showing that derivability in $G \Pi(\mathcal{C})$ implies derivability in $\Pi(\mathcal{C})$.

A closely related logic to Product Logic is the so-called Cancellative Hoop Logic [8], CHL for short. CHL is the $\overline{0}$-free fragment of Product Logic extended with the cancellation axiom

$$
(\varphi \rightarrow \varphi \& \psi) \rightarrow \psi
$$


Its algebraic semantics is given by the variety of cancellative hoops, whose linearly ordered elements are exactly Product chains without the 0 . CHL is complete with respect to the standard cancellative hoop, the real semi-open interval $(0,1]$ endowed with the restriction of Product Logic truth functions. For any countable Product subalgebra $\mathcal{C}$ of $[0,1]_{\Pi}$, one could define the logic $\mathrm{CHL}\left(\mathcal{C}^{-}\right)$, which is the expansion of CHL with truth-constants from $C^{-}=C \backslash\{0\}$, in a completely analogous way we have defined $\Pi(\mathcal{C})$ from $\Pi$. And moreover, a careful checking would show that all the results we have proved for $\Pi(\mathcal{C})$ could be easily transferred to $\mathrm{CHL}\left(\mathrm{C}^{-}\right)$.

As for future work, observe that, from the results in [10], it is possible to axiomatically define the logic of any particular continuous t-norm and its residuum. Then, the partial embeddability result, which now holds for the expansions of the three main continuous t-norms logics with truth-constants, could be extended to the expansion of any logic of a continuous t-norm with truth-constants. From there, we plan a general study of the expansions of any continuous t-norm logic with truth-constants.

It has been suggested by Petr Cintula (personal communication) to consider the extension of $\Pi(\mathcal{C})$ logics with the inference rule

$$
R_{r}: \text { from } \bar{r} \text { derive } \overline{0}
$$

for a particular $0<r<1$. Call the resulting logic $\Pi(\mathcal{C})^{\star}$. Notice that, since $\mathcal{C}$ is Archimedean, all the rules $R_{s}$, for any $0<s<1$, are derivable in $\Pi(\mathcal{C})^{\star}$. The consequence of the addition of such an inference rule is to discard all $\Pi(\mathcal{C})$-algebras of type II as possible algebraic models, as it happens in $\Pi_{\Delta}(\mathcal{C})$, since the rule forbids the collapsing of truth-constants. Therefore, it remains as an interesting task to study in more detail the logic $\Pi(\mathcal{C})^{\star}$, which is inbetween $\Pi(\mathcal{C})$ and $\Pi_{\Delta}(\mathcal{C})$, and whose class of corresponding algebras is not a variety but a quasivariety.

\section{Appendix: some results on ordered Abelian groups}

In this appendix we list some results on ordered Abelian groups that are used in the paper. Some of them are particular cases of most general results about ordered Abelian groups but we give the results we need and some of the proofs for the reader's convenience.

For the first result we recall the definition of the ordered group obtained as the lexicographic product of copies of the o.a.g of the positive real numbers with the natural order and the product operation $\left(\mathbb{R}^{+}, \cdot, \leq\right)$. For any natural $k$, we denote by $\left(\mathcal{R}^{+}\right)_{\text {lex }}^{k}=\left(\left(\mathbb{R}^{+}\right)^{k}, \bullet,(1, \ldots\right.$. $\left., 1), \leq_{\text {lex }}\right)$ the linearly ordered Abelian group defined on the Cartesian product of $k$ copies of the positive reals $\mathbb{R}^{+}$, with - being the coordinatewise multiplication and with $\leq_{\text {lex }}$ the lexicographic order. Note that the $\Rightarrow \bullet$ operation in the $\Pi$-algebra $\mathbf{P}\left(\mathcal{R}^{+}{ }_{\text {lex }}^{k}\right)$, with $(0, \ldots, 0)$ as bottom element, is defined as follows:

$$
\left(a_{1}, \ldots, a_{k}\right) \Rightarrow \bullet\left(b_{1}, . ., b_{k}\right)=\left\{\begin{array}{l}
(1, \ldots, 1), \text { if }\left(a_{1}, \ldots, a_{k}\right) \leq l e x\left(b_{1}, \ldots, b_{k}\right) \\
\left(1, \ldots, 1, b_{j} / a_{j}, \ldots, b_{k} / a_{k}\right), \text { otherwise }
\end{array}\right.
$$

where $j$ is the smallest index for which $a_{j}>b_{j}$.

The following result is a consequence of well-known Hahn's theorem, which is a more general result (see e.g. [13, Theorem 4.C]). However, a direct proof of the next theorem can be found in [15, Theorem 7.3.15].

Theorem 8.1. If $G$ is a finitely generated ordered Abelian group, then $G$ is isomorphic to a subgroup of $\left(\mathcal{R}^{+}\right)_{\text {lex }}^{k}$. 
The second result is given in the following lemma.

Lemma 8.2. Let $H$ be a subgroup of $\mathbb{R}^{+}$. Any function $t: H \cap(0,1] \rightarrow \mathbb{R}^{+}$such that $t(x \cdot y)=t(x) \cdot t(y)$ for all $x, y$ in $H \cap(0,1]$ may be extended to a group homomorphism $t^{\prime}: H \rightarrow \mathbb{R}^{+}$.

Proof. Define $t^{\prime}: H \rightarrow \mathbb{R}^{+}$as follows:

$$
t^{\prime}(x)= \begin{cases}t(x), & \text { if } x \leq 1 \\ 1 / t(1 / x), & \text { if } x>1\end{cases}
$$

For any $x, y \in H$, one (and only one) of the identities

$$
\begin{aligned}
t(x \cdot y) & =t(x) \cdot t(y) \\
t(x \cdot y) \cdot t(1 / x) & =t(y) \\
t(x \cdot y) \cdot t(1 / y) & =t(x) \\
t(1 / y) & =t(1 / x \cdot 1 / y) \cdot t(x) \\
t(1 / x) & =t(1 / x \cdot 1 / y) \cdot t(y) \\
t(1 / x) \cdot t(1 / y) & =t(1 / x \cdot 1 / y)
\end{aligned}
$$

is well defined and satisfied, and implies a corresponding identity with $t^{\prime}$ instead of $t$. Since for every $z \in H t^{\prime}(1 / z) \cdot t^{\prime}(z)=1$, we may derive $t^{\prime}(x \cdot y)=t^{\prime}(x) \cdot t^{\prime}(y)$ in each of the cases.

Finally the third result is a consequence of the fact that an Abelian group is injective if and only if it is divisible, see e.g. [18, Prop. 3, Sect. 4.2]. Since $\mathbb{R}^{+}$is abelian and divisible, the next lemma follows. For the reader's convenience, we provide a simple elementary proof of the particular case we need.

Lemma 8.3. Let $H$ be a subgroup of $\mathbb{R}^{+}, x \in \mathbb{R}^{+} \backslash H$ and let $H^{\prime}$ be the subgroup generated by $H$ and $x$. Then every homomorphism $t: H \rightarrow \mathbb{R}^{+}$may be extended to a homomorphism $t^{\prime}: H^{\prime} \rightarrow \mathbb{R}^{+}$.

Proof. If there is no $n \geq 1$ such that $x^{n} \in H$, then define $t^{\prime}(x)$ arbitrarily. Every element of $H^{\prime}$ has a unique decomposition as $x^{i} \cdot a$, where $i$ is an integer and $a \in H$, so we may define $t^{\prime}\left(x^{i} \cdot a\right)=t^{\prime}(x)^{i} \cdot t(a)$ and this yields a homomorphism on $H^{\prime}$.

If there is some $n \geq 1$ such that $x^{n} \in H$, denote by $n$ the smallest natural number with this property. For every integer $i$, we have $x^{i} \in H$ iff $n$ divides $i$. For every integer $i$ and every $a \in H$ define $t^{\prime}\left(x^{i} \cdot a\right)=t\left(x^{n}\right)^{i / n} \cdot t(a)$. Let us prove that this is a correct definition. If $x^{i} \cdot a=x^{j} \cdot b$ for integers $i, j$ and $a, b \in H$, then there is a natural $k$ such that $j=i-k \cdot n$ and $b=a \cdot x^{k n}$. It follows that $t\left(x^{n}\right)^{j / n} \cdot t(b)=t\left(x^{n}\right)^{i / n-k} \cdot t(a) \cdot t\left(x^{n}\right)^{k}=t\left(x^{n}\right)^{i / n} \cdot t(a)$. Moreover, the mapping $t^{\prime}$ is clearly a homomorphism on $H^{\prime}$. 


\section{Acknowledgments}

The authors thanks the anonymous reviewers for their helpful comments and suggestions. They are also indebted to Rostislav Horčík for his helpful comments and for pointing out the reference [13]. P. Savický acknowledges the support from the Czech Ministry of Education, Youth and Sports by the project 1M0021620808, Institute for Theoretical Computer Science - ITI. R. Cignoli, F. Esteva and L. Godo also acknowledge a bilateral Argentinian-Spanish project CONICET-CSIC, and F. Esteva and L. Godo a bilateral Czech-Spanish project CSAVCSIC. Finally, F. Esteva, L. Godo and C. Noguera also acknowledge partial support from the Spanish project TIN2004-07933-C0301.

\section{References}

[1] R. J. Adillon, V. Verdú. On product logic. Soft Computing 2, no. 3, 141-146, 1998.

[2] W. J. Blok and D. Pigozzi. Algebraizable logics. Mem. Amer. Math. Soc. 396, vol $77,1989$.

[3] R. Cignoli and D. Mundici. Partial isomorphisms on totally ordered abelian groups and Hájek's completeness theorem for Basic Logic, Multiple Valued Logic, special issue dedicated to the memory of Grigore Moisil, vol. 6 (2001) 89-94

[4] R. Cignoli And A. Torrens. An algebraic analysis of product logic. Mult. Val. Logic, 5 (2000), 45-65.

[5] P. Cintula. From Fuzzy Logic to Fuzzy Mathematics. Ph.D. dissertation, Czech Technical University, Prague (Czech Republic), 2005.

[6] P. Cintula. About axiomatic systems of product fuzzy logic. Soft Computing 5, (2001), no. $3,243-244$.

[7] F. Esteva and L. Godo. Monoidal t-norm based Logic: Towards a logic for leftcontinuous t-norms. Fuzzy Sets and Systems 124 (2001) 271-288.

[8] F. Esteva, L. Godo, P. Hásek, F. Montagna. Hoops and fuzzy logic. Journal of Logic and Computation 13 (2003), pp. 531-555.

[9] F. Esteva, L. Godo, P. Hájek, M. Navara. Residuated fuzzy logic with an involutive negation. Archive for Mathematical Logic 39 (2000) 103-124.

[10] F. Esteva, L. Godo And F. Montagna. The ŁП and ŁП $\frac{1}{2}$ logics: two complete fuzzy systems joining Łukasiewicz and Product logics. Archive for Mathematical Logic 40 (2001) 39-67.

[11] F. Esteva, L. Godo And C. Noguera. On Rational Weak Nilpotent Minimum Logics. To appear in Journal of Multiple-Valued Logic and Soft Computing. A preliminary version appeared in the Proc. of the Xth International Conference IPMU 2004, Perugia (Italia), July 4-9, 561-568, under the title "On Rational Gödel and Nilpotent Minimum logics."

[12] G. GeRla. Fuzzy Logic: Mathematical Tools for Approximate Reasoning. Trends in Logic 11, Kluwer, 2001. 
[13] A. M. W. GLass. Partially Ordered Groups. Series in Algebra, Vol. 7, World Scientific, 1999.

[14] P. HÁJEK. Metamathematics of Fuzzy Logic, Trends in Logic, vol.4 Kluwer, 1998.

[15] P. Hájek, T. Havránek, R. Jiroušek. Uncertainty Processing in Expert Systems. RC Press, Inc., Boca Raton, 1992.

[16] P. Hásek, L. Godo And F. Esteva. A complete many-valued Logic with Product conjunction. Archive of Mathematical logic 35 (1996) 19108.

[17] R. Horčí́k And P. Cintula. Product Łukasiewicz Logic. Archive for Mathematical Logic 43 (2004) 477-503.

[18] J. LAmbeK. Lectures on Rings and Modules, Blaisdel, Waltham, Massachusetts - Toronto - London, 1966.

[19] G. Metcalfe, N. Olivetti, D. Gabbay. Analytic Proof Calculi for Product Logics. Archive for Mathematical Logic 43(7), 859-889, 2004.

[20] F. Montagna, C. Noguera, R. HorČÍk. On weakly cancellative fuzzy logics. Submitted.

[21] V. NovÁk. On the syntactico-semantical completeness of first-order fuzzy logic. Part I. Syntax and Semantics.Kybernetika 26 (1990), 47-66.

[22] V. NovÁk. On the syntactico-semantical completeness of first-order fuzzy logic. Part II. Main Results.Kybernetika 26 (1990), 134-154.

[23] V. Novák, I. Perfilieva, J. MočKoř. Mathematical Principles of Fuzzy Logic. Kluwer Academic Pub., 1999.

[24] J. Pavelka. On Fuzzy Logic I, II, III. Zeitschrift fur Math. Logik und Grundlagen der Math. 25 (1979) 45-52, 119-134, 447-464.

[25] P. Vojtas. Fuzzy logic programming. Fuzzy Sets and Systems 124 (2001), 361-370. 